\title{
Quality Improvement in the NHS
}

\author{
Sahana Rao MBBS FRCPCH \\ Consultant Paediatrician, Oxford University Hospitals NHS trust \\ sahana.rao@ouh.nhs.uk
}

cite as: Rao S. (2018) Quality Improvement in the NHS. Sushruta 11(1) 17-18 DOI:

10.38192/11.1.6

Quality improvement (QI) is an integral part of providing high quality patient care. Any discussion about affordable healthcare cannot be complete without an affirmation to QI which needs to be a key priority. The resources in any health care setting including NHS are finite. In a changing medical landscape; while dealing with an increasing patient population; complex medical co- morbidities; ageing population and changing patient expectations, it is vital that we strive to continually use $\mathrm{QI}$ to improve our services.

\section{What is Ql?}

Quality improvement has been defined in various ways. "The degree to which health services for individuals and populations increase the likelihood of desired health outcomes and are consistent with current professional knowledge" (US Institute of Medicine). "The combined and unceasing efforts of everyone - healthcare professionals, patients and their families, researchers, payers, planners and educators- to make the changes that will lead to better patient outcomes (health), better system performance (care) and better professional development (learning)"(Paul Batalden and Frank Davidoff (BMJ Quality \& Safety 2007;16:23.)

\section{Why do we need to get involved with QI?}

Safe, Timely, Effective, Efficient, equitable and person-centred care are the six universal dimensions of healthcare quality. QI is essential for teams to ensure that patients get the right care in the right place at the right time, every time and improve efficiency of services. It is a key component of medical revalidation and GMC Good Medical Practice suggests that, "You must take steps to monitor and improve the quality of your work (13, Domain 1) and contribute to and comply with systems to protect patients and you must take part in systems of quality assurance and quality improvement to promote patient safety. (22, Domain 2: Safety and quality).

\section{Starting with QI: QI methods and tools}

A systematic narrative review of $\mathrm{Ql}$ models in healthcare (Powell et al, NHS Quality Improvement Scotland, 2009) concluded that "there is no one right method or approach that emerges above the others as the most effective".

Initiating change: 
The model for improvement provides a framework for developing, testing and implementing changes by answering the 3 key questions:

1. What are we trying to accomplish (aim)

2. How will we know a change is an improvement (measurement)

3. What changes we can make that will result in improvement.

Some methods to do this include root cause analysis; 5 whys; process mapping; trigger tools; mapping patient journey and patient \& staff experience data.

\section{Stakeholder engagement:}

Inspiring shared purpose and leading a team through a QI project can be challenging at times and having a team of resilient, passionate and committed enthusiasts is a battle half won. Patients, service users and their friends can make significant contributions to quality improvement projects by combining their perspective and expertise with those of staff involved in a service leading to robust and realistic proposals for change.

\section{Measurement of change}

Assessment and measurement are essential in QI. The data collected prior to the introduction of change can provide evidence that improvement is feasible. Regular measurements throughout the project will reveal if the QI work leads to the desired outcomes and if these improvements are sustained. Measurement can be both qualitative and quantitative. Outcome measures reveal the impact on patients; process measures reflect the systems, pathways and processes involved and balancing measures reflect the impact of the change on other areas. Run chart is an effective tool to collect, review and analyse the data. Small amounts of data are collected regularly and compiled into 'run charts' provides a pictographic representation of the impact of change over time.

\section{Implementing change:}

The Plan Do Study Act (PDSA) is a popular method for implementing change.

\section{Sustainability of a QI project:}

Irrespective of whether positive changes are achieved or not; it is essential to share the learning widely so that others can adopt it. There should be formalised processes in place to ensure the improvement is embedded into routine practice and sustained with governance arrangements.

\section{The future:}

The frontline NHS staff have an excellent understanding of the challenges in NHS and are motivated to lead change. Junior doctors are expected to undertake QI projects as part of their training and though motivated to participate in a high quality project; they may lack the skills to maximise the effectiveness. Formal support for developing quality improvement skills is often difficult to access and as a result quality improvement projects can be seen as a 'tick-box' exercise. In Oxford, we provide a comprehensive programme for QI in paediatrics with the QI teaching sessions; peer mentoring and resources to undertake QI projects. It is upto us to support our teams; equip them with the skills to channel their ideas; passion and enthusiasm and guide the next generation of NHS. 


\section{Bibliography}

https://www.ncbi.nlm.nih.gov/pubmed/25077248

https://qualitysafety.bmj.com/content/16/1/2

http://www.healthcareimprovementscotland.org/our_work/long_term_conditions/neurologica

1_health_services/neurological_standards_2009.aspx 